11,13

\title{
Неравновесная кинетика начальной стадии фазового перехода
}

\author{
() Г.И. Змиевская \\ Институт прикладной математики им. М.В. Келдыша РАН, \\ Москва, Россия \\ E-mail: zmig@mail.ru \\ Поступила в Редакцию 16 июля 2019 г. \\ В окончательной редакции 16 июля 2019 г. \\ Принята к публикации 25 июля 2019 г.
}

\begin{abstract}
Кинетические уравнения в частных производных Колмогорова-Феллера и Эйнштейна-Смолуховского с нелинейными коэффициентами решаются новыми устойчивыми численными методами. Теория стохастических динамических переменных устанавливает связь решения уравнений Ито в смысле Стратоновича для траекторий винеровских случайных процессов с плотностью переходной вероятности этих процессов, или функциями распределения кинетических уравнений. Классическая теория нуклеации (образования зародышей фазового перехода первого рода) описывает неравновесную стадию процесса конденсации диффузионным случайным процессом в пространстве размеров зародышей фазового перехода, когда флуктуации влияют на кластеризацию зародышей. Модель образования вакансионно-газовых дефектов (пор, блистеров) в кристаллической решетке, возникающих в результате ее облучения инертным газом $\mathrm{Xe}^{++}$, дополнена рассмотрением броуновского движения неточечных дефектов решетки, происходящего под действием суперпозиции парных дальнодействующих модельных потенциалов косвенного упругого взаимодействия пор между собой и с границами слоев. Пространственно-временные структуры пористости в образце формируются на временах порядка $10-100 \mu \mathrm{s}$ в результате броуновского движения вакансинногогазовых дефектов, для моделирования которого использованы устойчивые алгоритмы. По расчетам $10^{6}$ траекторий найдены неравновесные кинетические функции распределения пор по размерам и координатам в слоях облучаемых материалов, они характеризуют флуктуационную неустойчивость начальной стадии фазового перехода, по ним оцениваются локальные напряжения и пористость в модельном объеме.
\end{abstract}

Ключевые слова: кинетическая теория, уравнение Колмогорова, численные методы, флуктуации, неустойчивость, пористость, Броуновское движение, карбид кремния, блистеры.

DOI: $10.21883 /$ FTT.2020.01.48730.58ks

\section{1. Введение}

Классическая теория образования зародышей конденсации была развита в работах Фольмера, Беккера и Деринга, Зельдовича и Френкеля [1]; в случае конденсации пара образуются капли расплава или жидкости, при имплантации ионов инертного газа в кристаллическую решетку материала радиационные повреждения могут привести к формированию неточечных вакансионно-газовых дефектов (ВГД), или блистеров [2]. Расчеты вляния пор в твердом теле [3] на порог пластической деформации и возникновение напряжений в материалах принципиально при создании материалов с заданными свойствами [4] и для других приложений. Моделирование пористости в карбиде кремния востребовано, поскольку это алмазоподобный материал упрочняющих покрытиий, а также радиационно-стойкий широкозонный полупроводник [5]. Формирование пористости рассмотрим на начальной, неравновесной стадии фазового перехода первого рода, когда на изменение размера зародышей ВГД оказывают влияние флуктуации размеров, вызываемые неупругими столкновениями. Представим процессы образования зародышей фазового перехода первогого рода моделями случайных процессов, при этом для описания эволюции исполь- зуем теорию стохастических динамических переменных [6] (например, размера зародыша $g$ и его координат $\mathbf{r}=r(x, y, z)$ в объеме образца), $\{g(t), t \geq 0\}$, $\forall g \in\{\mathbf{G}\},\{r(t), t \geq 0\}, \forall r \in\{\mathbf{R}\}$, где $\{\mathbf{G}\}$ и $\{\mathbf{R}\}-$ фазовые пространства размеров зародышей и координат соответственно.Сформулированы кинетические уравнения в частных производных Колмогорова-Феллера [7] и Эйнштейна-Смолуховского с моделями нелинейных коэффициентов, что делает их квазилинейными, а дополнение уравнений источниками „мономеров“ (вакансий и частиц инертного газа), а также стоками отвечает приближению открытой физической системы Размер ВГД определяется кластеризацией вакансий и молекул инертного газа, а координаты ВГД изменяются в результате Броуновского движения (БД) кластеров ВГД [8].

Метод стохастической молекулярной динамики $[9,10]$ опирается на теоремы, согласно которым кинетические уравнения в частных производных параболического типа однозначно связаны со стохастическими дифференциальными уравнениями (СДУ) Ито, а плотность переходной вероятности марковского случайного процесса (МП), являющегося решением СДУ Ито, интерпретируется как функция распределения (ФР), входящая в соответствующее кинетическое уравнение [11]. 
Функционал-коэффициентами кинетических уравнений являются диффузия в пространстве размеров зародышей [10] и термодинамический потенциал, свободная энергия Гиббса, образования зародыша фазового перехода, в том числе и ВГД, в материале, модель кластеризации устанавливает нелинейную зависимость коэффициентов от размера зародыша $g$ и от упругих свойств материала, в решетке которого ВГД образуются. В течение неравновесной стадии фазового перехода постоянны энергия и доза потока инертного газа, то есть „мономеры“ имплантируемых частиц подобны пару в конденсации, пересыщение и давление по предположению постоянны, не меняется и температура образца из-за малой длительности стадии образования зародышей.

БД в кристаллических решетках материалов вызывается потенциалами косвенного упругого взаимодействия ВГД, которые являются броуновскими частицами с переменной массой. Зарождение газового пузырька в решетке возмущает колебания акустических фононов во всех материалах и фриделевские осцилляции электронной плотности в металлах и полупроводниках [12]. В случае системы с дискретными положениями ВГД и при малых их концентрациях важную роль играет дальнодействующая часть потенциала взаимодействия, в диэлектриках это взаимодействие является упругим, то есть косвенным взаимодействием через акустические фононы, в случае водорода в металле энергия взаимодействия порядка $0.01 \mathrm{eV}$ и при увеличении массы примеси может вырасти. В металлах более существенную роль играет другой вид дальнодействующего взаимодействия - косвенное взаимодействие через фриделевские осцилляции электронной плотности, величина которого составляет от $10^{-2}$ до $1 \mathrm{eV}$. При сближении дефектов надо учитывать короткодействующую часть потенциала экспоненциально убывающую с расстоянием между ВГД.

Функционал-коэффициентами в уравнении Смолуховского являются коэффициент диффузии и производная потенциала взаимодействия ВГД.

Термин „открытость“ моделируемых систем означает переменность числа частиц в расчетной области и/или несохранение полной энергии в системе за счет объемных источников и потоков через ее границы. Образование пористости (кластеров зародышей) на начальной стадии фазового перехода рассматривается как формирование долгоживущих (по сравнению с характерным временем $\tau_{r}=10^{-7} \mathrm{~s}$ для модели БД [11]) пространственно-временных образований в многомерных фазовых пространствах, подобно тому как были обнаружены в численных экспериментах когерентные структуры в фазовых пространствах сильно неравновесной плазмы [13].

Расчеты пористости, возникающей при внедрении ионов $\mathrm{Xe}^{++}$в материалы, методом стохастической молекулярной динамики рассматриваются с точки зрения модификации алгоритмов решения СДУ [14] и развития кинетической теории фазовых переходов [8-11].

\section{2. Кинетические уравнения и стохастические модели}

Кинетические уравнения [15] (Колмогорова-Феллеpa (1) для флуктуационного зародышеобразования и Эйнштейна-Смолуховского (2) для БД кластеров зародышей), после расщепления уравнения Колмогорова (основанного на различии характерных времен образования зародышей и их БД) выглядят следующим образом:

$$
\begin{gathered}
\frac{\partial f_{r}(g, t)}{\partial t}=\frac{1}{k T} \frac{\partial}{\partial g}\left[D_{g}(g, t) f_{r}(g, t) \frac{\partial \Delta \Phi(g, r, t)}{\partial g}\right] \\
+\frac{\partial}{\partial g}\left[D_{g}(g, t) \frac{\partial f_{r}(g, t)}{\partial g}\right]+S_{\alpha}, \\
f_{r}(g, 0)=f_{0 g},\left.\quad \frac{d f_{r}(g, t)}{d g}\right|_{g=2}=0,\left.\quad f_{r}(g, t)\right|_{g<2}=0 . \\
\frac{\partial f_{g}(\mathbf{r}, t)}{\partial t}=\frac{\partial}{\partial r}\left[D_{r}(\mathbf{r}, t) \frac{\partial f_{g}(\mathbf{r}, t)}{\partial r}\right] \\
-\frac{\partial}{\partial r}\left[\frac{F(\mathbf{r}, t)}{M_{g} \gamma} f_{g}(\mathbf{r}, t)\right]-Q, \\
\left.f_{g}(r, t)\right|_{x=x_{\text {left }}}=\left.f_{g}(r, t)\right|_{x=x_{\text {right }},}, \\
\left.f_{g}(r, t)\right|_{y=y_{\text {left }}}=\left.f_{g}(r, t)\right|_{y=y_{\text {right }}}, \\
\begin{array}{c}
N \\
\sum_{i \neq j} U_{i j}(\mathbf{r})+U_{\text {surf }}(\mathbf{r})+U_{p h}(\mathbf{r})+U_{\text {pore }}(\mathbf{r}),
\end{array}
\end{gathered}
$$

Здесь $S_{\alpha}\left(f_{\alpha}\right)$ - источник „мономеров“ (вакансий и/или газа $\mathrm{Xe}), f_{\alpha}-$ равновесная ФР имплантируемых частиц, в (1) $f_{r}(g, t)$ - кинетическая ФР кластеров зародышей газовых пузырьков (блистеров) по размерам в точке с координатой $r$, где $g$ - размер кластера в числе несжимаемых объемов вещества кластера. $D_{g}(g, t)$ - функционал-коэффициент диффузии в фазовом пространстве размеров кластеров $\{\mathbf{G}\}$, который зависит от $g$ как $D_{g}=D_{g 0} g^{2 / 3}$, а также от $k T$ и $p$ - температуры образца $(k-$ постоянная Больцмана) и давления „мономеров“ (по аналогии с давлением паров при конденсации); имплантацию потока ионов в материал характеризуют дозой, энергией ионов и флюенсом. $D_{g 0}-$ коэффициент модели. Функционал-коэффициент $\Delta \Phi(g, \mathbf{r})$ - свободная энергия Гиббса образования зародыша ВГД [16]. Полная свободная энергия Гиббса $\Delta \Phi$ образования ВГД в точке $r_{i}$ учитывает разность химических потенциалов фаз, вклад, пропорциональный поверхностному натяжению на границе „кластер-решетка“ с форм-фактором сферической формы ВГД, вклад в $\Delta \Phi$ от несоразмерности параметров решеток на границе слоев, энергия упругих связей решеток и условие разрыва их, положение ВГД в узлах - междоузлиях матрицы материала слоя. В уравнении (2) обозначены $M_{g}(g, \mathbf{r})=M_{g}\left(f_{r}(g, t), \mathbf{r}\right)$ - масса кластера, определяемая по ФР из решения (1), и $\gamma$ - коэффициент трения, сила $\mathbf{F}(\mathbf{r}, t)=\partial U(\mathbf{r}, t) / \partial \mathbf{r}$, 
где $U(\mathbf{r}, t)$ - дальнодействующий потенциал косвенного упругого взаимодействия пузырьков газа в решетке. ФР $f_{g}(\mathbf{r}, t)$ зависит от $\mathbf{r}-$ положения зародышей кластеров в ортогональной системе координат. Периодические граничные условия БД определены по границам области с координатами $X$ и $Y$; по направлению потока ионов $Z$ граничные условия задаются различно для облучаемой и необлучаемой границ; условие на границе между слоями требует более подробного описания. На верхней границе образца ставится условие нейтрализации потока ионов $\mathrm{Xe}^{++}$или источник частиц, поступающих в объем, задан равновесной функцией распределения имплантируемых ионов, отвечающей условиям „открытой физической системы“, также задаются дополнительные условия на осциллирующий дальнодействующий модельный потенциал взаимодействия ВГД, учитывающий взаимодействия: $U_{i j}(\mathbf{r})$ - „блистер-блистер“, $U_{s u r f}(\mathbf{r})$ - блистера с поверхностями, облучаемой ионами и разделяющей слои, $U_{p h}(\mathbf{r})$ - блистера с решеткой и $U_{\text {pore }}(\mathbf{r})-$ блистера с крупной порой.

Стохастические уравнения запишем для простоты без источника газа и вакансий, $S_{\alpha}=0$, также не конкретизируем стоки, $Q=0$. Тогда кинетическим уравнениям в частных производных Колмогорова-Феллера и Эйнштейна-Смолуховского для функций распределения $f_{r}(g, t)$ и $f_{g}(\mathbf{r}, t)$ будут соответствовать стохастические аналоги - уравнения для определения $r(t)$ и $g(t)$ Ито в смысле Стратоновича с приращениями винеровских случайных процессов, обозначенными $d w$ :

$$
\begin{gathered}
d r(t)=\left(\frac{F(r, t)}{M_{g} \gamma}-\frac{1}{2} \frac{\partial D_{r}(r, t)}{\partial r}\right) d t+\sqrt{2 D_{r}(r, t)} \circ d w(t) \\
d g(t)=\left(-\frac{D_{g}(g, t)}{k T} \frac{\partial \Delta \Phi(g, r, t)}{\partial g}-\frac{1}{2} \frac{\partial D_{g}(g, t)}{\partial g}\right) d t \\
+\sqrt{2 D_{g}(g, t)} \circ d w(t), \quad g(0)=g_{0}
\end{gathered}
$$

Система уравнений (3), (4) решается численным методом стохастической молекулярной динамики [10]. Стандартный расчет вариантов: количество траекторий составляет $10^{6}$, шаг по времени составляет $10^{-8} \mathrm{~s}=\tau_{g}$, количество шагов по времени составляет $10^{5}$. Физический процесс зарождения пор приводит к неустойчивости фазового перехода, его развитие численно моделируется алгоритмом, использующим устойчивые алгоритмы метода семейства Розенброка для решения СДУ в смысле Стратоновича [15], явного асимптотически несмещенного численного метода [14], который имеет второй порядок сходимости в среднеквадратичном пределе для системы СДУ с одиночным шумом или с аддитивными шумами. Узлы временной сетки $\left\{t_{n}\right\}$ включают однородную сетку с шагом $\tau_{g}=10^{-8} \mathrm{~s}$ для кластеризации и $\tau_{r}=10^{-7} \mathrm{~s}$ для БД по каждой из координат, СДУ для $\mathbf{r}=r(x, y, z, t)$ представлены в модели последовательно решаемыми одномерными задачами, время окончания расчета составляет $T_{f i n}=10^{4} \cdot \tau_{r}$. Схема позволяет нам моделировать решения СДУ, которые описывают физические процессы в различных масштабах характерных времен, среди которых: кластеризация пор в защитных покрытиях из карбида кремния и металла, а также БД кластеров в образце из двух слоев $(\mathrm{SiC} / \mathrm{Me}, \mathrm{Me}-$ металл).

Компьютерный эксперимент моделирования БД состоит из нескольких этапов. Начальное распределение координат ВГД задается, по нему рассчитывается распределение потенциала в объеме. Второй этап - расчет траекторий винеровских процессов (решение СДУ) как модели БД в решетке под воздействием коллективного потенциала взаимодействия ВГД, определяются значения стохастических динамических переменных в фазовом пространстве $\{\mathbf{R}\}$ координат ВГД. Далее определяется новое распределение значений потенциалов в области расчета и коэффициенты СДУ, зависящие от распределения дефектов по координатам.

Потенциал $U(x, y, z)$ является дальнодействующим и знакопеременным. Зависимости потенциалов взаимодействия от координат, в которых находятся ВГД, выведены с помощью диаграмм Фейнмана [12]; приведем пример потенциала взаимодействия „блистер-блистер“ для точечных масс

$$
\begin{aligned}
U_{i j}= & \sum_{i \neq j}^{N}\left(\frac{b_{r}\left[3 / 5-\frac{\left(x_{i}-x_{j}\right)^{4}+\left(y_{i}-y_{j}\right)^{4}+\left(z_{i}-z_{j}\right)^{4}}{\left(r_{i}-r_{j}\right)^{4}}\right]}{\left(r_{i}-r_{j}\right)^{3}}\right. \\
& \left.+\frac{a_{r} \cos \left(c_{r}\left|\mathbf{r}_{i}-\mathbf{r}_{j}\right|\right)}{\left(r_{i}-r_{j}\right)^{3}}\right),
\end{aligned}
$$

где $i, j$-- количество взаимодействующих ВГД, полагаемых сферами, если их нумеровать от 1 до $N$; коэффициенты $b_{r}, a_{r}, c_{r}$ - параметры модели решетки материала, знак потенциала зависит от комбинации ее упругих модулей, $U(x, y, z)$, считая поверхность Ферми (ПФ) сферической, нормирован на

$$
\left(\frac{N_{e} V_{F}\left(2 k_{F}\right)}{2 \pi \varepsilon^{2}}+W_{\text {elast }}\right) \frac{\Omega}{k T},
$$

где $\Omega$ - объем элементарной ячейки, $N_{e}-$ плотность состояний электронов на ПФ, $\varepsilon$ - диэлектрическая проницаемость как функция $k_{\mathrm{F}}, W_{\text {elast }}$ - модули упругости материала, $k_{\mathrm{F}}$ - фермиевский импульс электрона, $V_{\mathrm{F}}\left(2 k_{\mathrm{F}}\right)$ - фурье-компонента потенциала взаимодействия электрона с точечным дефектом.

Решение систем СДУ модифицированным [16] численным методом позволяет рассчитать ФР $f_{r}(g, t)$ и $f_{g}(x, y, z)$, отвечающие определению вероятностных $\Phi P$, найденных в вычислительном эксперименте, а также математическое ожидание и дисперсию значений стохастических динамических переменных и ФР сферических ВГД по размерам и координатам в объеме. 


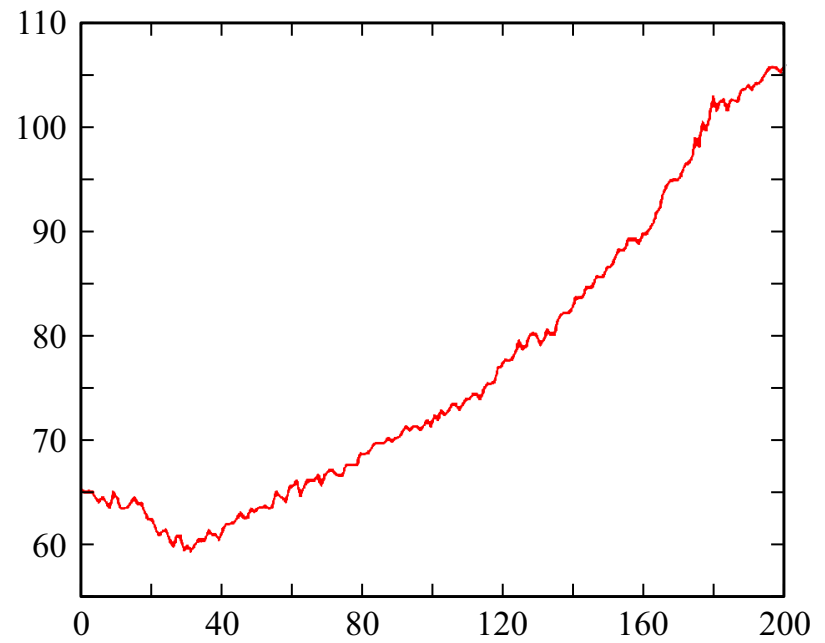

Рис. 1. Эволюция математического ожидания размера ВГд $\langle g\rangle=\langle g(t)\rangle=\int_{g=2}^{g \max } g f(g, t) d g$ (в числе несжимаемых объемов газа); безрезмерное время $-t / \tau$, где $\tau-$ шаг алгоритма $\left(\sim 10^{-8} \mathrm{~s}\right)$. ВДГ формируется в единице объема слоя $\mathrm{SiC}$ образца $\mathrm{SiC} /$ Мо с размерами $438 \times 438 \times 44 \mathrm{~nm}$ при значениях $T=1532 \mathrm{~K}$, энергии ионов $\mathrm{Xe}^{++} 4 \mathrm{keV}$ и дозе облучения $10^{16}$ ion $\cdot \mathrm{cm}^{-2}$; начальный вид ФР $f(g, t=0)$ задан так, что $\langle g(t=0)\rangle \sim g_{\text {crit }}, \frac{\partial \Delta \Phi\left(g_{\text {crit }}\right)}{\partial g}=0$, т. е. в области неустойчивости фазового перехода; область интегрирования в $\{G\}$ от $g_{\min }=2$ до $g_{\max }$.

Для расчета пористости $p_{j}$ в $j$-ом слое образца на глубине $z_{j}, j \in\left[z_{j}+\Delta z\right]$ используется формула:

$$
p_{j}=p\left(z_{j}, t\right)=\frac{\frac{4}{3} \pi r_{\mathrm{Xe}}^{3}}{N_{0} V_{l}} \sum_{i=1}^{N} g_{i}^{3} f\left(g_{i}, z_{j}, t\right),
$$

здесь $r_{\text {Хе }}-$ радиус объема ВГД газа Хе, $V_{l}-$ объем $j$-го слоя, $N_{0}$ - число ВГД при $t=0$. По неравновесным ФР могут быть найдены макроскопические характеристики материалов (такие как пористость, дисперсность, величина локального напряжения вблизи дефектов и др.).

\section{3. Пример расчета пористости}

Моделирование пористости в тонких слоях $\mathrm{SiC} / \mathrm{Me}$ было проведено для энергий ионов от 5 до $10 \mathrm{keV}$, при потоках инертного газа от $10^{15}$ до $10^{17} \mathrm{ion} / \mathrm{cm}^{2}$ и варьировании температуры образца. Каждый расчет проводился при постоянном значении характеристик потока и температуры субстрата. Решение уравнений в многомерных фазовых пространствах размеров кластеров и координат в самосогласованных дальнодействующих потенциалах позволяет визуализировать и структуру пористости, и неравновесное распределение кинетических ФР. Решение $10^{6}$ СДУ для $\{g(t), t \geq 0\}$ позволяет оценивать математическое ожидание среднего размера кластера зародыша (рис. 1). Сравнение с поверхностью равных значений ФР $f(g, z, t)$ (рис. 2) указывает на то, что ФР неравновесна и содержит намного больше информации о процессе образования ВГД, их распределение в слоях карбида кремния и Мо, которое при $t=0$ было задано равновероятными значениями по размерам и глубине, в конце расчета становится бимодальным, в защищаемый слой металла попадают мелкие дефекты и проникают до середины слоя металла. Пример расчета поверхности равных значений потенциала взаимодействия ВГД, под действием которого происходит БД, существенно зависит от концентрации дефектов, суперпозиция парных потенциалов взаимодействия ВГД приведена в сечении с координатой $z=Z_{0}$ (рис. 3 ) вид $U\left(x, y, z=Z_{0}\right.$ ) (в произвольных единицах на рисунке) приведен для двух слоев, учтен потенциал взаимодействия ВГД через акустические фононы и фриделевские осцилляции электронной плотности, на рис. 3 можно видеть влияние на $U(x, y, z)$ несоразмерности параметров решеток карбида кремния и металла. Распределение упругих сил в решетках слоев образца определяет БД и ФР координат

$a$

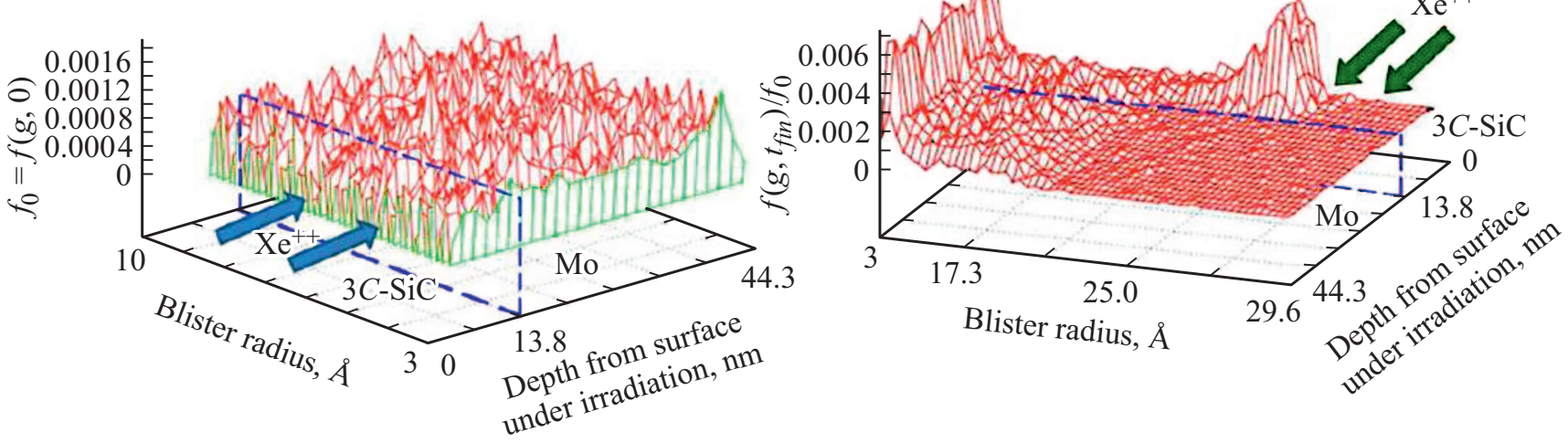

Рис. 2. ФР ВГД при тех же условиях, что и расчет $\langle g(t)\rangle$ (рис. 1 ); толщина слоя $3 C$-SiC -13.8 nт; начальное состояние задано равновероятным распределением $f_{0}=f(g, z, t=0)(a)$; в момент окончания расчета $t_{f i n}$ сформировалась неравновесная ФР, на рисунке приведена $f\left(g, z, t=t_{f i n}\right) / f_{0}$; размер ВГД $g$ и глубина образца $Z$ приведены в размерных единицах. 
пор (рис. 4), центры масс которых распределены в объеме двух слоев, визуализация заполнения порами объема слоев определяется перколяционным анализом координат ВГД и на рисунке приведена в сечениях простейшая диагностика распределения пор. Карта зарождения петель предтрещин относится к сечению двухслойного образца в слое молибдена, перпендикулярному падающему потоку, отстоящему от межслойной границы на пять параметров решетки. Белым цветом изображены вакансионно-газовые поры, остальное пространство соответствует материалу без дефектов, по ФР ВГД можно восстановить картину локальных напряжений в случае сфер и возникающие градиенты напряжений в процессе БД, а также оценить статистику размеров пространственно-временных объединений ВГД, расстояние между которыми задано условием принадлежности к „структуре“. Такой анализ зависимости наиболее вероятной протяженности структур в слое Мо от тем-

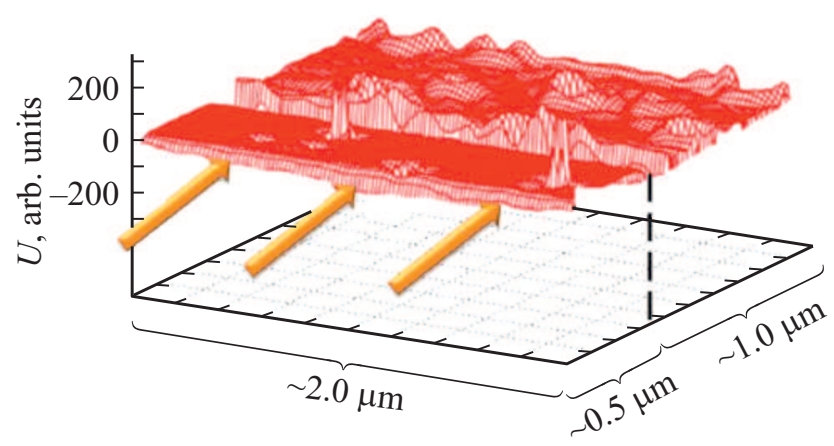

Pис. 3. Потенциал взаимодействия ВГД через акустические фононы и фриделевские осцилляции электронов в двухслойном образце $3 C$-SiC/Mo, полученный в ходе расчета образования заподышей фазового перехода в форме пор для образца с размерами $2 \times 2 \times 1.5 \mu \mathrm{m}$ с толщиной слоя $3 C$-SiC $0.5 \mu$ при облученном ионами ксенона с энергией $7 \mathrm{keV}$, доза облучения $10^{15} \mathrm{~cm}^{-2}$, при температуре образца $1500 \mathrm{~K}$. Потенциал построен в сечении расчетной области плоскостью XOZ.

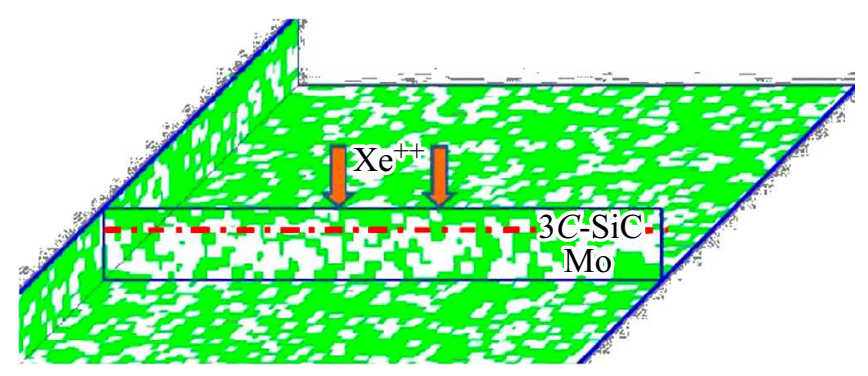

Рис. 4. Распределения ВГД в образце $3 C-\mathrm{SiC} / \mathrm{Mo}$ с размерами слоев, как на рис. 3, в произвольных сечениях относительно потока ионов; приводится рассчитанная по формуле (5) пористость; дополнительный перколяционный анализ распределения пор позволяет визуализировать изображение протяженных областей образца (структур ВГД). Области без пор закрашены, поры показаны белым цветом, горизонтальное сечение находится в слое Мо, условия те же, что на рис. 3.

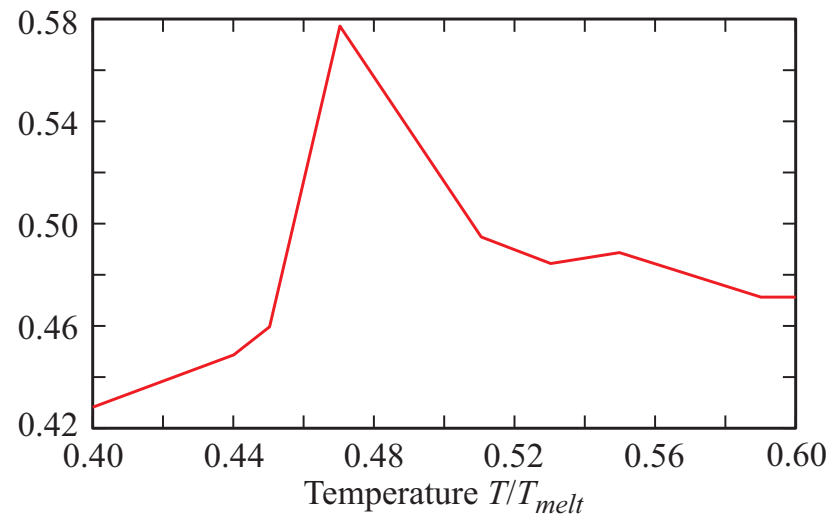

Рис. 5. Зависимость наиболее вероятного количества ВКД в протяженных структурах (количество ВГД в протяженных структурах отнесено к общему количеству ВГД в образце) от температуры в долях $T_{\text {melt }}$ температуры плавления наименее тугоплавкого материала $3 \mathrm{C}-\mathrm{SiC} / \mathrm{Mo}$,

пературы в интервале от 0.4 до $0.65 T_{\text {melt }}$, где $T_{\text {melt }}-$ температура плавления металла, приведен на рис. 5. Вычислительные эксперименты обращают внимание на нелинейную зависимость зарождения структур дефектов (предтрещин) от температуры, протяженность (количество ВГД) различно в направлении падающего потока (рис. 5) и в плоскостях, перпендикулярноых потоку $\mathrm{Xe}^{++}$, максимальные длины дефектов достигаются при существенно большей температуре образца $\left(0.57 T_{\text {melt }}\right)$.

\section{4. Заключение}

Компьютерный эксперимент расчета нестационарных ФР ВГД позволяет найти параметры пористости и напряжений в карбиде кремния $(3 C-\mathrm{SiC})$ как перспективном полупроводниковом материале и материале защитных покрытий под действием ионов инертного газа с энергиями в диапазоне до $10 \mathrm{keV}$. Определены кинетические ФР, формирующиеся в результате радиационностимулированного фазового перехода первого рода на флуктуационной стадии. По полученным в численном эксперименте неравновесным кинетическим ФР рассчитаны скорость зародышеобразования в неравновесных условиях, пористость, степень дисперсности и напряжения в слоях на временах порядка $1-100 \mu \mathrm{s}[8,10,11,16]$. Обнаружено, что средние размеры блистеров в обоих слоях подложки (и в металле, и в полупроводнике) нелинейно зависят от температуры. Найденное ранее свойство зависимости среднего размера блистеров от температуры прослеживается и в свойствах структур блистеров: максимальная длина протяженных структур дефектов нелинейно изменяется с изменением температуры слоев.

Фазовый переход в условиях открытой физической системы, например, при воздействии на вещество мощных источников энергии [17], как и при облучении ионами 
инертного газа тонкого слоя $\mathrm{SiC}$, становится неравновесным, при этом распределение зародышей новой фазы не будет стационарным, а будет изменяться с характерными временами свободного пробега первичных и вторичных частиц.

\section{Конфликт интересов}

Автор заявляет, что у него нет конфликта интересов.

\section{Список литературы}

[1] Я.Б. Френкель. Кинетическая теория жидкости. Изд-во АН СССР, М.-Л. (1945). 423 c.

[2] Н.В. Плешивцев, А.И Бажин. Физика воздействия ионных пучков на материалы. Вуз. кн. М. (1998). 392 с.

[3] В.В. Слезов, О.А. Осмаев, Р.В. Шаповалов. Движение пор в материале с источниками атомов газа. Вопросы атомной науки и техники, Сер. Физика радиационных повреждений и радиационное материаловедение 86, 3, 38 (2005).

[4] С.А. Кукушкин, А.В. Осипов. ФТТ 56, 761 (2014)

[5] Р.А. Андриевский. Основы наноструктурного материаловедения. Возможности и проблемы. БИНОМ Лаборатория знаний, М. (2012). 252 с.

[6] L. Arnold. Random dynamic system. Springer Monographs in Mathematics. Springer (1998). 586 p.

[7] Я.Б. Зельдович. ЖЭТФ 12, 525 (1942).

[8] G.I. Zmievskaya, A.L. Bondareva, V.D. Levchenko, T.V. Levchenko. J. Phys. D 40, 4842 (2007).

[9] Г.И. Змиевская. Физика плазмы 23, 368 (1997).

[10] A. Bondareva, T. Levchenko, G. Zmievskaya. Defect and Diffusion Forum. 297-301: Diffusion in Solids and Liquids V: 502. Trans Tech Publications, Switzerland (2010).

[11] G. Zmievskaya, T. Averina, A. Bondareva. Appl. Numerical Math. 15, 29, (2015).

[12] А.А. Берзин, А.И. Морозов, А.С. Сигов. ФТТ 14, 79 (1996).

[13] Ю.С. Сигов. Вычислительный эксперимент: мост между прошлым и будущим физики плазмы. Избр. тр. Сост. Г.И. Змиевская, В.Д. Левченко. Физматлит, М. (2001). $288 \mathrm{c}$.

[14] S.S. Artem'ev, T.A. Averina. Numerical analysis of systems of ordinary and stochastic differential equations. VSP The Netherlands, Utrecht (1997). 176 p.

[15] S.W. Gardiner. Handbook of Stochastic Methods for Physics, Chemistry, and the Natural Sciences. Springer, Berlin (1984). $440 \mathrm{p}$.

[16] Г.И. Змиевская, А.Л. Бондарева. Поверхность. Рентгеновские, синхротронные и нейтронные исследования 8,33 (2016).

[17] Ф.Х. Мирзоев, В.Я. Панченко, Л.А. Шелепин. УФН 166, 3 (1996).

Редактор Е.Ю. Флегонтова 